\title{
Procedimentos dolorosos e medidas de alívio em Unidade de Terapia Intensiva Neonatal*
}

\author{
Painful procedures and relieving \\ measures in NICU
}

\author{
Fernando de Souza Nóbrega ${ }^{1}$, Lígia Sakai², \\ Vera Lúcia Jornada Krebs ${ }^{3}$
}

\begin{abstract}
Nóbrega FS, Sakai L, Krebs VLJ. Procedimentos dolorosos e medidas de alívio em Unidade de Terapia Intensiva Neonatal. Rev Med (São Paulo). 2007 out.-dez.;86(4):201-6.

RESUMO: Objetivo: Descrever os procedimentos dolorosos e as medidas de alívio em recémnascidos internados em UTI neonatal. Métodos: estudo prospectivo, com dados obtidos por consulta ao prontuário, durante a internação de 52 neonatos admitidos em UTI, de maio a agosto de 2005. Foram descritos os procedimentos dolorosos e as medidas de alívio para cada situação. As variáveis qualitativas foram representadas por suas freqüências relativas e absolutas e as quantitativas por média, mínimo e máximo. Resultados: O tempo médio de internação foi 20 dias. Os diagnósticos mais freqüentes foram síndrome do desconforto respiratório, malformação digestiva, prematuridade e sepse. Foram realizados 1549 procedimentos dolorosos (média por paciente=30) e medidas de alívio foram tomadas em $56,7 \%$ destes. Os procedimentos mais freqüentes foram venóclise $(41,4 \%)$ e coleta de sangue $(21,1 \%)$. Nestas situações foram usados analgésicos em cerca da metade dos casos. Os medicamentos mais utilizados foram a dipirona e o fentanil. Medidas não medicamentosas não foram utilizadas. Conclusões: Os procedimentos dolorosos foram freqüentes durante a internação, sendo cada neonato submetido, em média, a 30 procedimentos. Medidas de alívio para dor foram utilizadas em $56,7 \%$ dos procedimentos, enquanto $43,3 \%$ dos mesmos foram realizados sem analgesia. Os medicamentos, principalmente dipirona e fentanil, foram a única forma de tratamento da dor. Sugerimos ampliar o uso de medidas analgésicas no tratamento destes neonatos, destacando a importância da prevenção das repercussões tardias da dor experimentada nesta faixa etária. Medidas simples não medicamentosas, como o uso de solução glicosada por via oral ou leite materno, podem ser utilizadas como estratégia adicional.
\end{abstract}

DESCRITORES: Recém-nascido. Dor. Analgesia. Unidades de Terapia Intensiva Neonatal.

\footnotetext{
* Pesquisa realizada como bolsista de Iniciação Científica (PIBIC) com bolsa concedida pelo Depto. de Pediatria da FMUSP.

1. Aluno do $4^{\circ}$ ano do curso de graduação em Medicina da FMUSP.

2. Aluna do $4^{\circ}$ ano do curso de graduação em Medicina da FMUSP.

3. Livre-docente do Depto. de Pediatria da FMUSP, Chefe do Berçario Anexo à Maternidade do HCFMUSP.

Endereço para correspondência: Rua Jaupaci, 629. CEP: 02318-150. São Paulo, SP. e-mail: fernando_nobrega@ hotmail.com
} 


\section{INTRODUÇÃO}

$\mathrm{N}$ os últimos 25 anos houve grande progresso no conhecimento sobre os mecanismos da dor e a resposta ao estímulo nociceptivo no recém-nascido (RN). Até então, os neonatos eram considerados incapazes de responder aos estímulos dolorosos, devido à imaturidade orgânica. Conseqüentemente, não eram utilizadas práticas adequadas para o tratamento da dor em Unidades de Terapia Intensiva neonatal3,15,16. $\mathrm{Na}$ última década, estudos sobre a fisiologia da dor demonstraram que o recém-nascido apresenta os componentes anatômicos e funcionais necessários para a apreciação do estímulo doloroso. Sabe-se que o recém-nascido possui um sistema nociceptivo intacto e funcional, sendo a densidade de terminações nervosas nociceptivas na pele do recém-nascido semelhantes ou até superiores àquelas observada na pele do adulto ${ }^{4}$

As respostas fisiológicas do neonato à dor incluem aumento da freqüência cardíaca, freqüência respiratória, pressão intracraniana, sudorese e diminuição da saturação da hemoglobina pelo oxigênio e do tônus vagal $\left.\right|^{9,10,13}$. A resposta comportamental caracteriza-se por reflexo de retirada, chutes, movimentos corpóreos, choro agudo e caretas. $\mathrm{Na}$ presença de dor e estresse crônico observa-se passividade, diminuição dos movimentos corporais, face inexpressiva, alterações da freqüência cardíaca e respiratória e do consumo de oxigênio ${ }^{1,5}$. Anand et al. ${ }^{3}$, chamaram a atenção para a maior incidência de hemorragia intraventricular e alterações isquêmicas levando a leucomalácia periventricular em recémnascidos pré-termo submetidos a estímulos dolorosos.

Os avanços recentes na área de terapia intensiva neonatal possibilitaram aumento da sobrevida de recém-nascidos extremamente prematuros e/ou gravemente doentes. Por outro lado, o tratamento dessas crianças inclui um grande número de procedimentos dolorosos ${ }^{8}$. Apesar do conhecimento sobre os efeitos imediatos e tardios da dor experimentada pelo RN, as medidas de alívio ainda não são realizadas rotineiramente nas unidades neonatais ${ }^{14}$. Esse fato pode ser explicado, em parte, pelo desconhecimento dos profissionais de saúde sobre o tema ${ }^{6,11}$.

Nossa hipótese é que o conhecimento da natureza dos procedimentos dolorosos e das medidas de alívio utilizadas em UTI neonatal possa contribuir para aperfeiçoar as estratégias de humanização no atendimento dessas crianças. O objetivo do presente estudo foi descrever os procedimentos dolorosos e as medidas de alívio em recém-nascidos internados em
UTI neonatal durante o período de quatro meses.

\section{CASUÍSTICA E MÉTODOS}

Foram incluídos prospectivamente no estudo 52 recém-nascidos admitidos na Unidade de Cuidados Intensivos Neonatal (UCINE) do Instituto da Criança do Hospital das Clínicas da Faculdade de Medicina da Universidade de São Paulo, no período de 11 de maio a 11 de agosto de 2005. Foram descritos os procedimentos dolorosos e as medidas de alívio para cada situação.

Os dados foram obtidos por consulta ao prontuário médico, durante a internação do paciente. Em todos os recém-nascidos os procedimentos foram identificados conforme o registro diário no prontuário, bem como as medidas de alívio relacionadas a cada procedimento.

Foram considerados procedimentos dolorosos: coleta de sangue, punção vesical, dissecção venosa, cateterização umbilical, drenagem torácica, coleta de líquido cefalorraquidiano (LCR), aspiração traqueal, intubação e passagem de sonda gástrica. Foram consideradas medidas de alívio: glicose oral antes e após procedimento, anestésico tópico, medicamento oral (paracetamol, dipirona, outros) e medicamento endovenoso (fentanil, morfina, outros).

Para todos os pacientes foi preenchido um protocolo contendo identificação, diagnóstico, procedimentos dolorosos e medidas de alívio.

Para caracterizar a amostra estudada foi feita análise descritiva dos dados. As variáveis qualitativas foram apresentadas em suas freqüências relativas (percentuais) e absolutas $(\mathrm{N})$ de cada classe de variável. As variáveis quantitativas foram representadas por média, mínimo e máximo.

O estudo foi aprovado pela Comissão de Pesquisa e Ética do Departamento de Pediatria da FMUSP e pela comissão de Ética para Análise de Projetos de Pesquisa do HC-FMUSP.

\section{RESULTADOS}

No período do estudo foram admitidos 52 neonatos na UCINE. Houve 27 (52\%) do sexo masculino e 25 (48\%) do sexo feminino. Todos os recém-nascidos foram submetidos a pelo menos um procedimento doloroso durante a internação. A idade gestacional variou de 24 semanas a 40,3 semanas (média $=35,4$ semanas) e o peso de nascimento variou de $720 \mathrm{~g} \mathrm{a}$ $4250 \mathrm{~g}$ (média $=2419,7 \mathrm{~g}$ ). Houve $17(36,2 \%$ ) recémnascidos pré-termo e $30(63,8 \%)$ recém-nascidos de termo. O tempo de internação variou de 1 dia a 97 dias $($ média $=20$ dias, Tabela 1 ). 
Tabela 1. Estatística descritiva do peso de nascimento, idade gestacional e duração da internação em 52 recém-nascidos admitidos em Unidade de Cuidados Intensivos Neonatal

\begin{tabular}{llll}
\hline & Mínimo & Máximo & Média \\
\hline Peso de Nascimento (g) & 720 & 4250 & 2420 \\
Idade Gestacional (semanas) & 24 & 40,3 & 35,3 \\
Duração da Internação (dias) & 1 & 97 & 20,2 \\
\hline
\end{tabular}

Os diagnósticos e a internação estão apresentados na Tabela 2. Foram realizados 1549 procedimentos considerados dolorosos (Tabela 3), sendo que as medidas de alívio foram indicadas em 36 (70\%) recém-nascidos. Todas as medidas de alívio foram de natureza medicamentosa (Tabela 4).

Tabela 2. Diagnósticos à internação em 52 recém-nascidos admitidos em Unidade de Cuidados Intensivos Neonatal

\begin{tabular}{lll}
\hline Diagnóstico à internação & $\mathrm{n}$ & $\%$ \\
\hline Síndrome do Desconforto Respiratório & 14 & 26,9 \\
Malformação digestiva & 10 & 19,2 \\
Prematuridade & 10 & 19,2 \\
Sepse & 8 & 15,4 \\
Icterícia & 8 & 15,4 \\
Baixo Peso & 8 & 15,4 \\
Bronquiolite & 5 & 9,6 \\
Febre sem sinais localizatórios & 5 & 9,6 \\
Cardiopatia congênita & 4 & 7,7 \\
Hipertensão pulmonar & 3 & 5,8 \\
Hipoglicemia & 3 & 5,8 \\
Risco infeccioso & 2 & 3,8 \\
Asfixia perinatal & 2 & 3,8 \\
Sífilis congênita & 2 & 3,8 \\
Linfangioma cervical & 1 & 1,9 \\
Meningite & 1 & 1,9 \\
Fenda palatina & 1 & 1,9 \\
Hidrocefalia & 1 & 1,9 \\
Infeção urinária & 1 & 1,9 \\
Anemia & 1 & 1,9 \\
Hiponatremia & 1 & 1,9 \\
Broncopneumonia & 1 & 1,9 \\
Síndrome convulsiva & 1 & 1,9 \\
Síndrome de Down & 1 & 1,9 \\
\hline
\end{tabular}

Tabela 3. Procedimentos dolorosos e medidas de alívio em 52 recém-nascidos admitidos em Unidade de Cuidados Intensivos Neonatal

\begin{tabular}{lrcc}
\hline Procedimento & $\mathrm{N}(\%)$ & medidas de alívio N(\%) & № de procedimentos/paciente \\
\hline Venóclise & $641(41,4 \%)$ & $324(50,5 \%)$ & 12,33 \\
Coleta de sangue & $327(21,1 \%)$ & $172(52,6 \%)$ & 6,29 \\
Passagem de sonda gástrica & $242(15,6 \%)$ & $137(56,6 \%)$ & 4,65 \\
Punção de calcanhar para glicemia & $183(11,8 \%)$ & $75(41,0 \%)$ & 3,52 \\
Intubação traqueal & $29(1,9 \%)$ & $21(72,4 \%)$ & 0,56 \\
Coleta de liquor & $29(1,9 \%)$ & $15(51,7 \%)$ & 0,56 \\
Cateter de inserção periférica (PICC) & $18(1,2 \%)$ & $8(44,4 \%)$ & 0,33 \\
Dissecção venosa & $17(1,1 \%)$ & $17(100 \%)$ & 0,31 \\
Cirurgia & $16(1,0 \%)$ & $16(100 \%)$ & 0,27 \\
Punção vesical & $14(0,9 \%)$ & $5(35,7 \%)$ & 0,23 \\
Passagem de sonda retal & $12(0,8 \%)$ & $12(100 \%)$ & 0,15 \\
Retirada de coletor de urina & $8(0,5 \%)$ & $3(37,5 \%)$ & 0,08 \\
Passagem de sonda vesical & $4(0,3 \%)$ & $3(75 \%)$ & 0,06 \\
Cateter umbilical & $3(0,2 \%)$ & $3(100 \%)$ & 0,04 \\
Drenagem torácica & $2(0,1 \%)$ & $2(100 \%)$ & 0,04 \\
Broncoscopia & $2(0,1 \%)$ & $2(100 \%)$ & 0,02 \\
Laringoscopia & $1(0,1 \%)$ & $1(100 \%)$ & 0,02 \\
Drenagem de abscesso & $1(0,1 \%)$ & $0(0 \%)$ & 29,8 \\
TOTAL & 1549 & $816(52,7 \%)$ & \\
\hline
\end{tabular}


Nóbrega FS. Procedimentos dolorosos e medidas de alívio em UTI Neonatal.

Tabela 4. Medidas de alívio durante procedimentos dolorosos em 52 recém-nascidos admitidos em Unidade de Cuidados Intensivos Neonatal

\begin{tabular}{lll}
\hline Medida de alívio & $\mathrm{N}$ & $\%$ \\
\hline Dipirona & 28 & 53,8 \\
Fentanil & 16 & 30,8 \\
Paracetamol & 4 & 7,7 \\
Anestesia geral & 9 & 17,3 \\
Anestesia local & 6 & 11,5 \\
Nenhuma & 16 & 30,8 \\
\hline
\end{tabular}

\section{DISCUSSÃO}

Constatamos a realização de 1549 procedimentos em 52 neonatos, durante o tempo médio de internação de 21 dias. Este resultado concorda com outros autores que chamam a atenção para o elevado número de estímulos dolorosos sofridos pelo recém-nascido durante sua permanência na $\mathrm{UTI}^{7,12}$. As medidas de alívio foram tomadas em $69,2 \%$ dos pacientes internados no período do estudo e estiveram ausentes em $30,8 \%$ dos casos, mostrando que um terço dos pacientes não foi tratado para a dor. Consideramos expressiva a porcentagem de pacientes submetidos a procedimentos dolorosos sem qualquer tipo de analgesia.

Em relação ao número de procedimentos, constatamos que em $56,6 \%$ destes foram utilizados analgésicos, enquanto em $43,3 \%$ não foram tomadas medidas para aliviar a dor. Os procedimentos mais freqüentemente realizados foram venóclise $(41,4 \%)$ e coleta de sangue $(21,1 \%)$. Nestas situações foram prescritos analgésicos em cerca da metade dos casos (Tabela 3).

Este resultado concorda com vários estudos que demonstram a importância da avaliação da dor no neonato e a subutilização de estratégias para o alívio da mesma. No Brasil, Prestes et al..$^{14}$, em quatro unidades de terapia intensiva neonatal, relataram não ter sido administrada nenhuma medicação específica para o alívio da dor aguda durante a intubação traqueal, punção vascular e punção lombar. Na inserção de dreno de tórax, $100 \%$ dos recém-nascidos receberam analgesia específica e, para a passagem de cateteres centrais, apenas $8 \%$. O medicamento mais utilizado foi o fentanil (93\%). Em nossa casuística, o uso sistemático de analgesia ocorreu somente para a realização de procedimentos cirúrgicos de grande porte, como cirurgia digestiva, ou de pequeno porte (dissecção venosa).

Observamos em nossa casuística que o único tipo de tratamento da dor foi medicamentoso, sendo os medicamentos mais utilizados a dipirona e o fentanil. Intervenções simples, como o uso de glicose por via oral ou aleitamento materno previamente à realização do procedimento, não foram realizadas em nenhum paciente. Esse fato pode ser explicado, em parte, pela gravidade das patologias apresentadas pelos recém-nascidos. Por outro lado, alguns estudos comprovaram a eficácia analgésica de medidas não medicamentosas, como a ingestão de glicose ou leite materno e a sucção não nutritiva durante a internação na terapia intensiva, em recém-nascidos que podem ser alimentados por via oral ${ }^{11}$.

A Academia Americana de Pediatria ${ }^{2}$, recomenda as seguintes diretrizes para o alívio da dor no recém-nascido: as medidas e métodos disponíveis para avaliar a dor devem ser usados consistentemente, enquanto o recém-nascido necessitar de tratamento; os profissionais de saúde devem usar apropriadamente medidas ambientais, comportamentais e farmacológicas para prevenir, reduzir ou eliminar o estresse e dor no neonato; devem ser utilizados agentes farmacológicos com propriedades farmacocinéticas e farmacodinâmicas conhecidas e com eficácia demonstrada em neonatos; agentes que alterem a função cardio-respiratória devem ser usados somente por profissionais com experiência em manejo das vias aéreas e em locais com condições de monitorização contínua; as instituições de saúde devem desenvolver e implementar políticas de cuidados ao paciente para o manejo e prevenção da dor em neonatos, inclusive naqueles recebendo cuidados paliativos; devem ser desenvolvidos programas educacionais para aumentar a habilidade dos profissionais de saúde no manejo do estresse e da dor; devem ser desenvolvidos e aprovados métodos para avaliação da dor que sejam facilmente aplicáveis no acompanhamento clínico; na realização de pesquisas deve haver uma padronização mínima para as medidas de controle da dor, a fim de permitir a análise estatística adequada.

A pouca utilização de medidas de alívio para a dor em recém-nascidos tem sido atribuída, em parte, ao desconhecimento dos profissionais de saúde da resposta ao estímulo nociceptivo no período neonatal. Chermont et al. ${ }^{7}$, entre 104 pediatras de Unidades de Terapia Intensiva e berçários, que responderam a um questionário sobre o conhecimento dos métodos de avaliação e tratamento da dor no período neonatal obtiveram dados relevantes. Foi constatado que, embora $100 \%$ dos profissionais acreditassem que o recém-nascido sente dor, somente um terço dos mesmos conheciam algum tipo de método de avaliação do estímulo nociceptivo nesta faixa etária. O uso de medidas analgésicas no pós-operatório de cirurgia abdominal foi relatado em menos da metade dos entrevistados e a referência de utilização de analgesia 
para punções venosas e capilares foi inferior a $10 \%$. Debillon et al. ${ }^{8}$ analisaram o tratamento da dor em Unidades de Terapia Intensiva neonatais na França através de questionários, demonstrando que dois terços das unidades francesas utilizam escores para dor no período neonatal. Os fatores associados com falha na utilização foram Unidades de Terapia Intensiva de nível de atendimento secundário, hospitais não universitários, pacientes clínicos e atendimento exclusivo de neonatos. Os autores destacaram que a educação sobre o manejo da dor é mais efetiva se o alvo for a enfermagem nas Unidades de Terapia Intensiva neonatais com as características listadas acima. Houve grande variabilidade na abordagem farmacológica, sugerindo a necessidade de elaboração de um consenso internacional de dor e realização de novos estudos randomizados.

Lago et al. ${ }^{9}$ destacam que a falta de tempo para administrar drogas durante procedimentos de emergência, a relação risco/benefício do uso de drogas para procedimentos muito breves em crianças sob risco de efeitos adversos e a baixa confiança em tais medicamentos são justificativas comuns para explicar a falha no controle da dor. A falta de treinamento na avaliação da dor e dificuldades objetivas devido à ausência de comunicação verbal clara são outros fatores que contribuem para o tratamento insuficiente da mesma em recém-nascidos. Embora medidas farmacológicas e não farmacológicas seguras já estejam bem estabelecidas, mesmo para recém-nascidos pré-termo, e escalas de dor válidas ou indicadores comportamentais e fisiológicos sejam disponíveis, estes recursos não são rotineiramente utilizados na maioria das UTI neonatais e variam consideravelmente, dependendo da situação clínica.

Concluímos que os procedimentos dolorosos foram freqüentes durante a internação, sendo as medidas de alívio insuficientes. Os medicamentos, principalmente dipirona e fentanil, foram a única forma de tratamento da dor. Sugerimos a ampliação do uso de medidas analgésicas em recém-nascidos no tratamento destes neonatos, destacando a importância da prevenção das repercussões tardias da dor experimentada nesta faixa etária. Medidas simples não medicamentosas, como o uso de solução glicosada por via oral ou leite materno, podem ser utilizadas como estratégia adicional.

Nóbrega FS, Sakai L, Krebs VLJ. Painful procedures and relieving measures in NICU. Rev Med (São Paulo). 2007 out.-dez.;86(4):201-6.

\begin{abstract}
S: Objective: To describe the painful procedures and relieving measures in newborns admitted at the Neonatal Intensive Care Unit (NICU). Methods: Prospective study of 52 neonates admitted at the NICU from May to August 2005; data were obtained from the patient's files. The painful procedures and the respective pain-relieving measures are described. The qualitative variables were represented by their relative and absolute frequencies and the quantitative variables, by means, minimum and maximum values. Results: Mean hospital stay duration was 20 days. The most frequent diagnoses were respiratory distress syndrome, digestive malformation, prematurity and sepsis. 1,549 painful procedures were performed (mean per patient $=30$ ) and relieving measures were carried out in $56.7 \%$ of these. The most frequent procedures were venoclysis (41.4\%) and blood collection (21.1\%). Analgesia was used in around half of the cases. The most commonly employed analgesic drugs were dipyrone and fentanyl. Non-medicamentous measures were not employed. Conclusions: Painful procedures were frequent during the hospital stay, and each neonate was submitted, on average, to 30 procedures. Pain-relieving measures were employed in $56.7 \%$ of the procedures, whereas $43.3 \%$ of them were carried out with no analgesia. Medicamentous measures, mainly dipyrone and fentanyl, were the only form of pain relief. We suggest increasing the use of analgesic measures in these neonates in order to treat the pain, and emphasized the importance of the prevention the late pain-related outcomes experienced in this age range. Simple measures such as oral glucose solution or breast milk can be used as an additional strategy.
\end{abstract}

KEY WORDS: Infant, newborn. Pain. Analgesia. Intensive Care Units, Neonatal.

\title{
REFERÊNCIAS
}

1. Abud-Saad HH, Bours GW, Stevens B, Hamers JPH. Assessment of pain in the neonate. Semin Perinatol. 1998;22:402-16.
2. American Academy of Pediatrics. Canadian Pediatric Society: prevention and management of pain and stress in the neonate. Pediatrics. 2000;105:454-61. 
Nóbrega FS. Procedimentos dolorosos e medidas de alívio em UTI Neonatal.

3. Anand KSS. Clinical importance of pain and stress in preterm neonates. Biol. Neonate. 1998;73:1-9.

4. Anand KSS, Phil D, Carr DB. The neuroanatomy, neurophysiology and neurochemistry of pain, stress and analgesia in newborns and children. Pediatr Clin. North Am. 1989;35:795-822.

5. Anand KSS, Phil D, Hickey PR. Pain and its effects in the human neonatal and fetus. $\mathrm{N}$ Engl $\mathrm{J}$ Med. 1987;317:1321-9.

6. Balda RCX, Guinsburg R, Almeida MFB, Peres CA, Miyoshi $\mathrm{MH}$, Kopelman BI. The recognition of facial expression of pain in full-term newborn by parents and health professionals. Arch Pediatr Adolesc Med. 2000;154:1009-16.

7. Chermont AG, Guinsburg R, Balda RCX, Kopelman $B I$. What do pediatricians know about pain assessment and treatment in newborn infants? J Pediatr. (RJ). 2003;79(3):265-72.

8. Debillon T, Bureau V, Savagner C, Zupan-Simunek V, Carbajal R. Pain management in French neonatal intensive care units. Acta Paediatr. 2002;91:822-6.

9. Lago P, Guadagni A, Merazzi D, Ancora G, Bellieni CV, Cavazza A. Pain management in the neonatal intensive care unit: a national survey in Italy. Pediatr Anesthesia.
2005; 15:925-31.

10.Larsson BA. Pain management in neonates. Acta Paediatr. 1999;88:1301-10.

11.Larsson BA, Tannfeldt G, Laggrcrantz H, Olsson GL. Veinpuncture is more effective and less painful than heel lancing for blood tests in neonates. Pediatrics. 1998;101:882-6.

12.Ldh V, Wiklund U, Hakansson S. Assessment of the effect of EMLA®during venipuncture in the newborn by analysis of heart rate variability. Pain. 2000;86:24754.

13. Porter FL, Wolf CM, Gold J, Lotsoff D, Miller JP. Pain and pain management in newborn infants: a survey of physicians and nurses. Pediatrics. 1997;100:626-32.

14. Prestes ACY, Guinsburg R, Balda RCX, Marba STM, Rugolo LMSS, Pachi PR. The frequency of pharmacological pain relief in university neonatal intensive care units. J Pediatr. (RJ). 2005;81:405-10.

15. Shah VS, Taddio A, Bennett S, Speidel BD. Neonatal pain response to heel stick us venepuncture for routine blood sampling. Arch Dis Child Fetal. 1997;77:F143-4.

16. Stevens B, Gibbins S, Franck LS. Treatment of pain in the neonatal intensive care unit. Pediatr Clin North Am. 2000;47:633-51. 